\title{
IMAGE QUALITY IMPROVEMENT USING IMAGE PRO- CESSING METHOD IMAGE BRIGHTNESS CONTRAST AND IMAGE SHARPENING
}

\author{
Fitri Rizani ${ }^{1 *}$ \\ ${ }^{1}$ Universitas Almuslim \\ *Corresponding author E-mail: fitri.asykar@gmail.com
}

\begin{abstract}
Keywords:
Image Brightness; Image Sharpening;

Image Processing; Histogram;

Image Quality

Abstract

The ability of computers that are increasingly reliable in various fields, especially in helping the image processing sector through improving image quality, is very much felt so that the empowerment of computers at any time needs to be improved. Image quality improvement can be made with various techniques, including Image Quality Improvement with Image Brightness and Image Sharpening methods. The process begins with capturing the image and then continues with increasing the intensity of brightness, image contrast and sharpening. Image processing results are indicated by changes in the resulting image and changes in the image histogram.
\end{abstract}

\section{Introduction}

The ability of computers that are increasingly reliable in various fields[1], especially in assisting the field of digital image processing through improving image quality, is immense so that the empowerment of computers is always being improved. With the support of software and hardware, and other devices, computers can help solve humans' problems[2]. Image is an image in a two-dimensional plane. Image processing is a system where the process is carried out with image input, and the result is also an image. Because the digital image is different from the analogue image to achieve the image change process, a calculation technique for RGB values and methods of calculating the mind is needed. Improving image quality is one of the initial processes in improving image quality[3]. Improving image quality is necessary because the image that is the object of discussion has poor quality[4]; for example, the image experiences noise, blurring, and so on.

The contrast image brightness method is made in several steps[5], namely image enhancement, in an image repair process by increasing the image quality[6], both contrast and brightness. Image restoration, improving an image model, colour image processing involves a colour image, either in image enhancement, image restoration or others. And many other techniques[7]. One of them is the brightness method in the computerized search system and the sharpness method in the image with a separate calculation method with the assumption of a matrix that is then calculated and replaced by the RGB value of the image to achieve an implementation of the method. Image enhancement is a process of obtaining images that are easier for the human eye to interpret. Image processing[8], which is included in image quality improvement, consists of processes that aim to improve image quality to obtain image beauty, image analysis purposes[9], and correct images. From the description above, the writer will develop the knowledge that the author has so far obtained in lectures, with the title: "Image Quality Improvement Using Image Processing Method Image Brightness Contrast and Image Sharpening".

\section{Method}

In this study, 2-dimensional still images are used, represented in a matrix with the size of $\mathrm{N} \times \mathrm{M}$. The matrix can also be considered a 2 dimensional array or a 2-dimensional array because it has 2 image coordinates, namely the $\mathrm{X}$ coordinate and the $\mathrm{Y}$ coordinate. Conventional 2-dimensional definition of arrays can lead to a waste of memory[10]. Image representation using a vector system is more relevant than a matrix structure. In this case, vectors are used to manipulate an image in a matrix (2-dimensional array) into a 1-dimensional array[11]. Using this technique, an object or image can be considered a 1-dimensional array of a vector. Therefore we need the appropriate 
data type. The data type used to handle vector systems is the pointer data type PbyteArray. Using this data type, the maximum amount of memory will be dynamic, according to the size of the image being used.

RGB is a colour model consisting of red, green, and blue, combined to form a wide colour array. Each base colour, for example, red, can be assigned a value range. For a computer monitor, the value ranges from the smallest $=0$ and the largest $=255$. This 256 scale choice is based on expressing the 8 digit binary number used by a computer machine. In this way, you will get a mix of $256 \times 256 \times 256$ $=1677726$ types of colours. A colour type can be thought of as a vector in 3-dimensional space, which is usually used in mathematics; the coordinates are expressed in three numbers: the $\mathrm{x}$-component, the $\mathrm{y}$-component and the $\mathrm{z}$-component. Suppose a vector is written as $\mathrm{r}$ $=(\mathrm{x}, \mathrm{y}, \mathrm{z})$. For colour, these components are replaced by the components $\mathrm{R}$ (Red), $\mathrm{G}$ (Green), B (Blue). A colour type can be written as follows: colour $=\operatorname{RGB}(30,75,255)$. White $=\operatorname{RGB}(255,255,255)$, while for black $=\operatorname{RGB}(0,0,0)$. For example: there is an image measuring 100 pixels x 100 pixels with a color encoding of 24 bits with $R=8$ bits, $G=8$ bits, $B=8$ bits, then the color encoding will be able to represent $0 \ldots 16,777,215$ (representing 16 million colors), and required disk space $=100 \times 100 \times 3$ bytes (due to RGB) $=30,000$ bytes $=30 \mathrm{~KB}$ or $100 \times 100 \times 24$ bits $=240,000$ bits.

\subsection{Analysis of Image Brightness Method}

The digital image can be defined as a function of two variables, $f(x, y)$, where $x$ and $y$ are spatial coordinates and the value $f(x, y)$ is the intensity of the image at these coordinates, this is illustrated in Figure 3.2 Basic technology for creating and displaying Color in digital images is based on the research that colour is a combination of three basic colours, namely red, green, and blue (Red, Green, Blue RGB). Colour images are more common images. The colour seen in the bitmap image is a combination of three basic colours, namely red, green, and blue. Each pixel is composed of three colour components: R (red), G (green), B (blue). The combination of the three RGB colours produces a unique colour for the corresponding pixel. In a 256 colour image, each pixel is 8 bits long, but its RGB colour components are stored in an RGB table called a palette. RGB is a colour model consisting of red, green, and blue, combined to form a wide colour array. Each base colour, for example, red, can be assigned a value range.

For computer monitors, the range should be at least $=0$ and maximum $=255$. This 256 scale option is based on expressing the 8 digit binary number used by computer machines. In this way, you will get a mix of 256 x 256 x $256=1677726$ types of colours. To be able to find out the RGB value in the image in figure 2, the author makes a feature in the new system to be able to find out the R, G and B values in the image, as for the system that can take these values can be explained in the following flowchart:

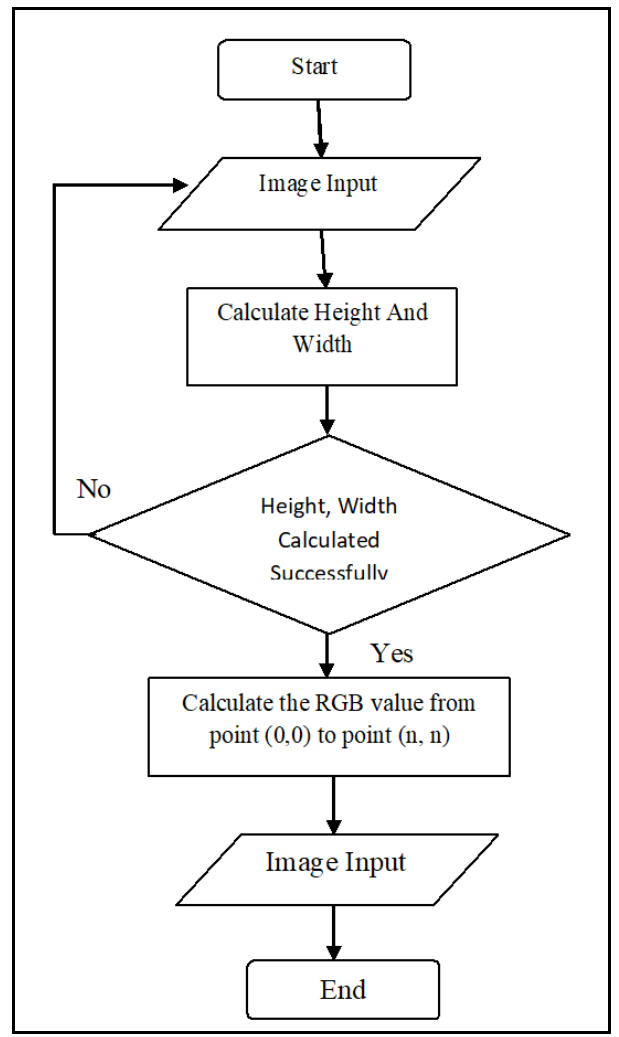

Fig. 1: RGB Calculation System Flowchart

The results of the calculation of the system in figure 2 below show RGB values for manual calculations in Table 1 and Table 2 with the provisions of the individual method.

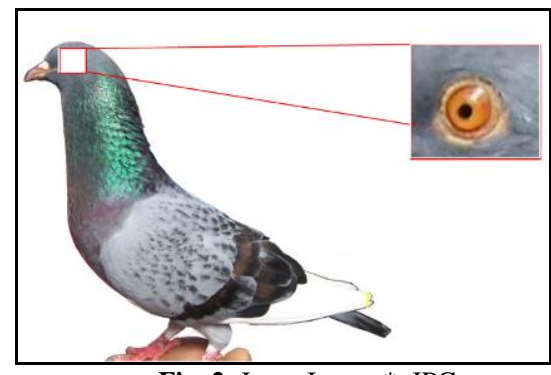

Fig. 2: Input Image *.JPG 
From the input image above, the RGB value of the cut part of the image is taken, as shown in figure 2 with the following image resolution:

Height $($ Height $)=111$

Weight $($ Width $)=97$

To adjust the brightness of the image in Figure 2 above, the brightness level of an image can be expressed in the formula:

$\mathrm{U}^{\prime}=\mathrm{U}+\mathrm{c}$

Where :

$\mathrm{U}$ 'and $\mathrm{U}=$ images after and before brightness adjustment

$\mathrm{C}=$ constant of adjustment factor

Then the results of enlightening the image in figure 2 above are as follows:

$\mathrm{U}$ is the RGB value of each pixel then added with a constant brightness intensity value of 10; the result is as shown in the table below: So that the RGB value table can be formed as follows, with a sample of RGB values of some image coordinate points:

Table 1: Analysis Table of Brightness Method

\begin{tabular}{|c|c|c|c|c|c|c|}
\hline \multirow[t]{2}{*}{$\begin{array}{l}\text { Coordinate point } \\
(\mathrm{x}, \mathrm{y})\end{array}$} & \multicolumn{3}{|c|}{$\begin{array}{l}\text { ORIGINAL IMAGE COL- } \\
\text { OUR }\end{array}$} & \multicolumn{3}{|c|}{ BRIGHTNESS INTENSITY=10 } \\
\hline & $\mathrm{R}$ & G & B & $\mathrm{R}$ & $\mathrm{G}$ & B \\
\hline$(3,110)$ & 189 & 146 & 112 & 199 & 156 & 122 \\
\hline$(3,111)$ & 160 & 111 & 78 & 170 & 121 & 88 \\
\hline$(3,112)$ & 180 & 127 & 95 & 190 & 137 & 105 \\
\hline$(3,113)$ & 218 & 158 & 130 & 228 & 168 & 140 \\
\hline$(3,114)$ & 224 & 160 & 135 & 234 & 170 & 145 \\
\hline$(3,115)$ & 197 & 131 & 109 & 207 & 141 & 119 \\
\hline$(3,116)$ & 159 & 90 & 74 & 169 & 100 & 84 \\
\hline$(3,117)$ & 170 & 102 & 89 & 180 & 112 & 99 \\
\hline$(3,118)$ & 201 & 139 & 126 & 211 & 149 & 136 \\
\hline$(3,119)$ & 241 & 183 & 171 & 251 & 193 & 181 \\
\hline$(3,120)$ & 255 & 201 & 189 & 265 & 211 & 199 \\
\hline$(3,121)$ & 233 & 183 & 172 & 243 & 193 & 182 \\
\hline$(3,122)$ & 202 & 152 & 141 & 212 & 162 & 151 \\
\hline$(3,123)$ & 197 & 149 & 139 & 207 & 159 & 149 \\
\hline$(3,124)$ & 191 & 144 & 134 & 201 & 154 & 144 \\
\hline$(3,125)$ & 173 & 131 & 119 & 183 & 141 & 129 \\
\hline$(3,126)$ & 173 & 135 & 124 & 183 & 145 & 134 \\
\hline$(3,127)$ & 167 & 133 & 121 & 167 & 143 & 131 \\
\hline$(3,128)$ & 155 & 127 & 115 & 165 & 137 & 125 \\
\hline$(3,129)$ & 155 & 131 & 121 & 165 & 141 & 131 \\
\hline$(3,130)$ & 141 & 121 & 114 & 151 & 131 & 124 \\
\hline$(3,131)$ & 114 & 99 & 94 & 124 & 109 & 104 \\
\hline$(3,132)$ & 96 & 86 & 84 & 106 & 96 & 94 \\
\hline$(3,133)$ & 91 & 85 & 85 & 101 & 95 & 95 \\
\hline$(3,134)$ & 96 & 92 & 93 & 106 & 102 & 103 \\
\hline$(3,135)$ & 103 & 99 & 100 & 113 & 109 & 110 \\
\hline$(3,136)$ & 95 & 89 & 89 & 105 & 99 & 99 \\
\hline$(3,137)$ & 77 & 68 & 69 & 87 & 78 & 79 \\
\hline$(3,138)$ & 67 & 57 & 56 & 77 & 67 & 87 \\
\hline$(3,139)$ & 69 & 59 & 58 & 79 & 69 & 68 \\
\hline$(3,140)$ & 72 & 62 & 62 & 82 & 72 & 72 \\
\hline$(3,141)$ & 68 & 58 & 57 & 78 & 68 & 67 \\
\hline$(3,142)$ & 56 & 46 & 47 & 66 & 56 & 57 \\
\hline$(3,143)$ & 48 & 39 & 40 & 58 & 49 & 50 \\
\hline$(3,144)$ & 47 & 41 & 41 & 57 & 51 & 51 \\
\hline$(3,145)$ & 61 & 57 & 58 & 71 & 67 & 68 \\
\hline$(3,146)$ & 72 & 67 & 71 & 82 & 77 & 81 \\
\hline$(3,147)$ & 67 & 65 & 70 & 77 & 75 & 80 \\
\hline$(3,148)$ & 63 & 62 & 68 & 73 & 72 & 78 \\
\hline$(3,149)$ & 59 & 58 & 64 & 69 & 68 & 74 \\
\hline$(3,150)$ & 58 & 57 & 62 & 68 & 67 & 72 \\
\hline$(3,151)$ & 71 & 71 & 73 & 81 & 81 & 83 \\
\hline$(3,152)$ & 34 & 34 & 34 & 44 & 44 & 44 \\
\hline$(3,153)$ & 75 & 74 & 72 & 85 & 84 & 82 \\
\hline$(3,154)$ & 254 & 253 & 249 & 255 & 255 & 255 \\
\hline$(3,155)$ & 255 & 254 & 250 & 255 & 255 & 255 \\
\hline$(3,156)$ & 251 & 249 & 249 & 255 & 255 & 255 \\
\hline$(3,157)$ & 249 & 249 & 249 & 255 & 255 & 255 \\
\hline$(3,158)$ & 250 & 250 & 252 & 255 & 255 & 255 \\
\hline
\end{tabular}


After the image brightness process is carried out with a light intensity of 10, an image that looks brighter can be produced as shown below:

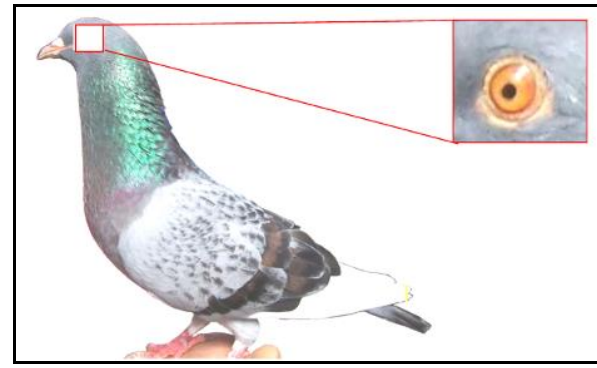

Fig. 3: Image Brightness Result

\subsection{Analysis of the Sharpness Method}

The image sharpness method can be analyzed on the intensity of the RGB value in the previous input image. From the input image above, the RGB value of the part of the image that has been cut is taken, as shown in Figure 3 with the following image resolution:

Height $($ Height $)=111$

Weight (Width) $=97$

To adjust the brightness of the image in Figure 3 above, the brightness level of an image can be expressed in the formula:

if $U$ '> 128 then

$\mathrm{U}^{\prime}=\mathrm{U}-(\mathrm{c} / 5)$

if $U$ ' $<128$ then

$\mathrm{U}^{\prime}=\mathrm{U}+(\mathrm{c} / 5)$

Provided that if $\mathrm{R}, \mathrm{G}, \mathrm{B}>255$ then $\mathrm{R}, \mathrm{G}, \mathrm{B}=255$

if $\mathrm{R}, \mathrm{G}, \mathrm{B}<0$ then $\mathrm{R}, \mathrm{G}, \mathrm{B}=0$

Where :

$\mathrm{U}$ 'and $\mathrm{U}=$ images after and before brightness adjustment

$\mathrm{C}=$ constant of adjustment factor

Then the results of enlightening the image in figure 3 above are as follows:

$\mathrm{U}$ is the RGB value of each pixel then added with a constant brightness intensity value of 10 ; the results are as shown in the table below: So that the RGB value table can be formed as follows, with a sample of RGB values of some image coordinate points:

Table 2: Analysis Table of the Sharpness Method

\begin{tabular}{|c|c|c|c|c|c|c|}
\hline \multirow[t]{2}{*}{$\begin{array}{c}\text { Coordinate point } \\
(\mathrm{x}, \mathrm{y})\end{array}$} & \multicolumn{3}{|c|}{$\begin{array}{l}\text { ORIGINAL IMAGE COL- } \\
\text { OUR }\end{array}$} & \multicolumn{3}{|c|}{ BRIGHTNESS INTENSITY $=10$} \\
\hline & $\mathrm{R}$ & $\mathrm{G}$ & B & $\mathrm{R}$ & $\mathrm{G}$ & B \\
\hline$(3,110)$ & 189 & 146 & 112 & 187 & 144 & 114 \\
\hline$(3,111)$ & 160 & 111 & 78 & 158 & 113 & 80 \\
\hline$(3,112)$ & 180 & 127 & 95 & 178 & 113 & 80 \\
\hline$(3,113)$ & 218 & 158 & 130 & 216 & 156 & 97 \\
\hline$(3,114)$ & 224 & 160 & 135 & 222 & 158 & 133 \\
\hline$(3,115)$ & 197 & 131 & 109 & 195 & 129 & 111 \\
\hline$(3,116)$ & 159 & 90 & 74 & 157 & 92 & 76 \\
\hline$(3,117)$ & 170 & 102 & 89 & 168 & 104 & 91 \\
\hline$(3,118)$ & 201 & 139 & 126 & 199 & 137 & 128 \\
\hline$(3,119)$ & 241 & 183 & 171 & 239 & 181 & 169 \\
\hline$(3,120)$ & 255 & 201 & 189 & 253 & 199 & 187 \\
\hline$(3,121)$ & 233 & 183 & 172 & 231 & 181 & 169 \\
\hline$(3,122)$ & 202 & 152 & 141 & 200 & 150 & 139 \\
\hline$(3,123)$ & 197 & 149 & 139 & 195 & 147 & 137 \\
\hline$(3,124)$ & 191 & 144 & 134 & 189 & 142 & 132 \\
\hline$(3,125)$ & 173 & 131 & 119 & 171 & 129 & 117 \\
\hline$(3,126)$ & 173 & 135 & 124 & 171 & 133 & 122 \\
\hline$(3,127)$ & 167 & 133 & 121 & 165 & 131 & 119 \\
\hline$(3,128)$ & 155 & 127 & 115 & 153 & 129 & 117 \\
\hline$(3,129)$ & 155 & 131 & 121 & 153 & 129 & 123 \\
\hline$(3,130)$ & 141 & 121 & 114 & 139 & 123 & 116 \\
\hline$(3,131)$ & 114 & 99 & 94 & 116 & 101 & 96 \\
\hline$(3,132)$ & 96 & 86 & 84 & 98 & 86 & 86 \\
\hline$(3,133)$ & 91 & 85 & 85 & 93 & 87 & 87 \\
\hline$(3,134)$ & 96 & 92 & 93 & 98 & 94 & 95 \\
\hline$(3,135)$ & 103 & 99 & 100 & 105 & 101 & 102 \\
\hline$(3,136)$ & 95 & 89 & 89 & 97 & 91 & 91 \\
\hline$(3,137)$ & 77 & 68 & 69 & 79 & 70 & 71 \\
\hline$(3,138)$ & 67 & 57 & 56 & 69 & 59 & 58 \\
\hline$(3,139)$ & 69 & 59 & 58 & 71 & 61 & 60 \\
\hline$(3,140)$ & 72 & 62 & 62 & 75 & 65 & 65 \\
\hline$(3,141)$ & 68 & 58 & 57 & 70 & 60 & 59 \\
\hline
\end{tabular}




\begin{tabular}{|c|l|l|l|l|l|l|}
\hline$(3,142)$ & 56 & 46 & 47 & 58 & 48 & 49 \\
\hline$(3,143)$ & 48 & 39 & 40 & 50 & 41 & 42 \\
\hline$(3,144)$ & 47 & 41 & 41 & 49 & 43 & 43 \\
\hline$(3,145)$ & 61 & 57 & 58 & 63 & 59 & 60 \\
\hline$(3,146)$ & 72 & 67 & 71 & 79 & 69 & 73 \\
\hline$(3,147)$ & 67 & 65 & 70 & 69 & 67 & 72 \\
\hline$(3,148)$ & 63 & 62 & 68 & 65 & 64 & 70 \\
\hline$(3,149)$ & 59 & 58 & 64 & 61 & 60 & 66 \\
\hline$(3,150)$ & 58 & 57 & 62 & 60 & 59 & 64 \\
\hline$(3,151)$ & 71 & 71 & 73 & 73 & 73 & 36 \\
\hline$(3,152)$ & 34 & 34 & 34 & 36 & 76 & 36 \\
\hline$(3,153)$ & 75 & 74 & 72 & 77 & 251 & 74 \\
\hline$(3,154)$ & 254 & 253 & 249 & 252 & 252 & 247 \\
\hline$(3,155)$ & 255 & 254 & 250 & 253 & 247 & 248 \\
\hline$(3,156)$ & 251 & 249 & 249 & 249 & 247 & 247 \\
\hline$(3,157)$ & 249 & 249 & 249 & 247 & 248 & 247 \\
\hline$(3,158)$ & 250 & 250 & 252 & 248 & & 250 \\
\hline
\end{tabular}

After the image brightness process is carried out with a light intensity of 10, an image that looks brighter can be produced as shown below:

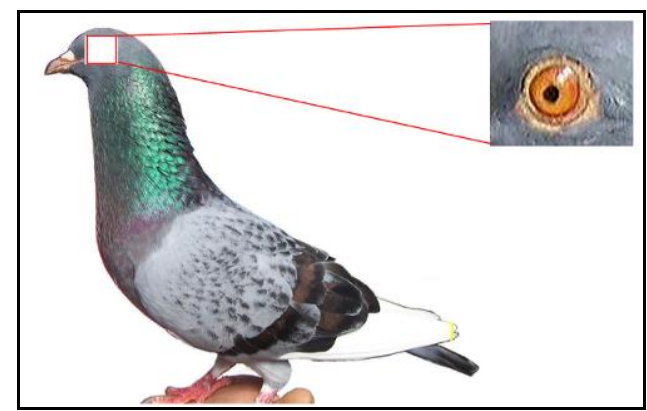

Fig. 4: Sharpness Result Image

\section{Results and Discussion}

\subsection{Test}

The test is carried out functionally. The method used in this test is a test that focuses on the functional requirements of the software being built.

\subsection{Program Praise}

At this stage, the application of the interface design results is carried out into a system built using the software described in the software implementation sub-chapter. Following the author's discussion, following the desired test, here are some views of image processing applications using the sharpness and sharpness method.

RGB information menu, this information menu is in the same form as the form that displays the image results, RGB information will appear after the get RGB button is clicked, the RGB value is the RGB value before being given enlightenment and sharpness in the image.

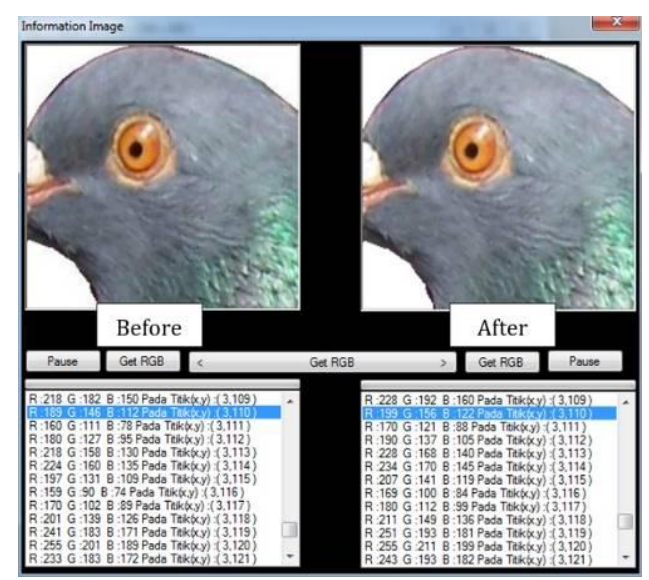

Fig. 5: Sharpness Result Image

The histogram is a graph depicting the distribution of pixel intensity values. This analysis will compare the original image histogram with the image histogram resulting from the brightness method. The histogram has many benefits in image processing, including deter- 
mining digitization parameters and selecting threshold limits. The peaks of the histogram show the intensity of the protruding pixels. The width of the peaks indicates the range of image contrast. An image that has a lighter or too dark contrast has a narrow histogram.

The histogram appears to use only half the degree of a grey area. A good image has a histogram that fills the full degree of a grey area with an even distribution of each pixel intensity. Table 2 shows the comparison of the original image histogram and the image produced by the Brightness method.

Table 3: Histogram Image

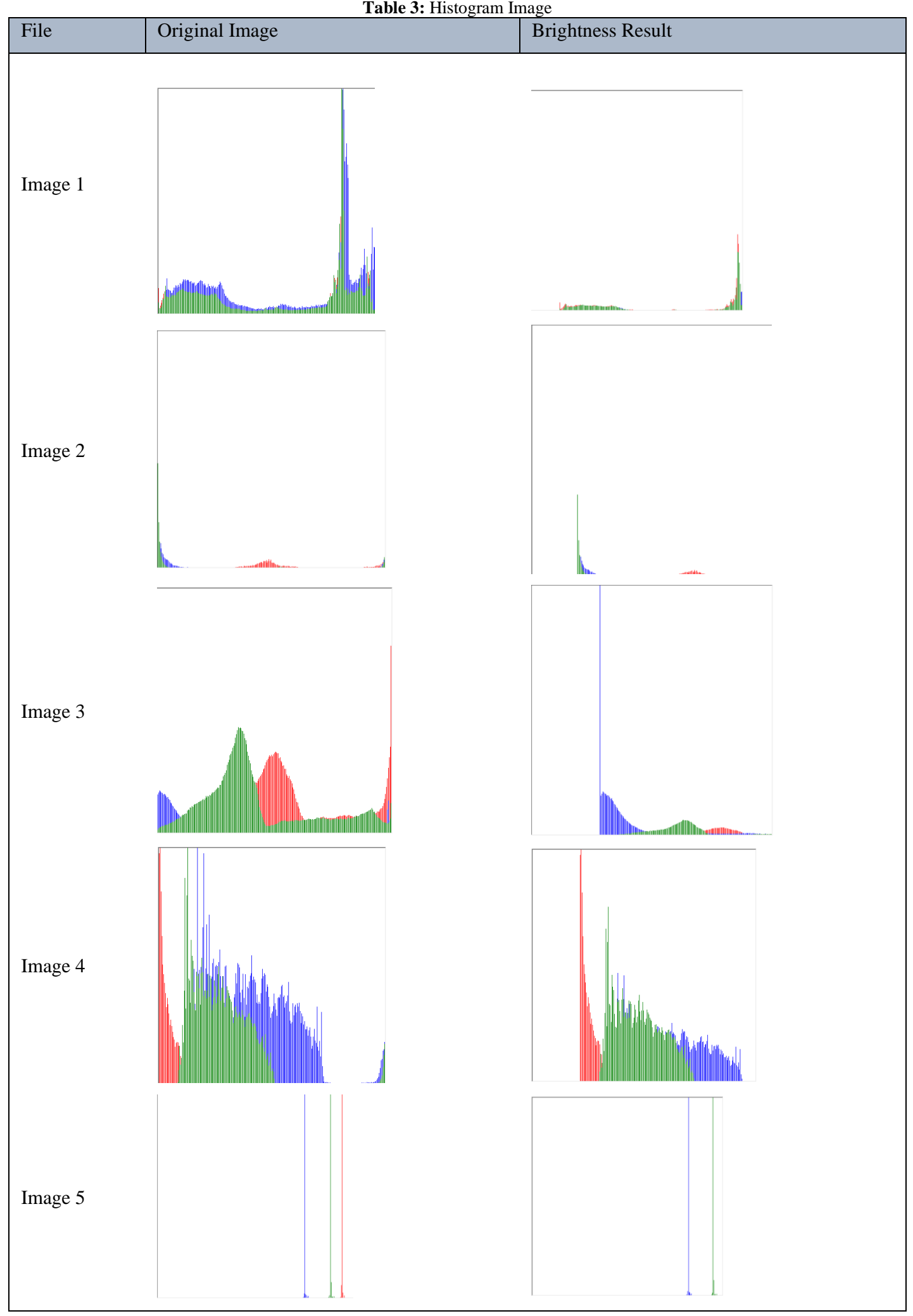

\section{Conclusion}

The following are some suggestions and suggestions related to image processing applications using the enlightenment and sharpness methods.

- Image processing is one of the steps to change the basic image into a better image. 
- The brightness method helps process the image in increasing the lighting of the image.

- The sharpness method is beneficial in processing the image to smooth the image pixels.

- Microsoft Visual Studio 2005 is an application that can build application programs with several programming languages with a specific project.

- Image processing application program built by the author can apply enlightenment and sharpness using the input image selected by the user via explorer browse.

In general, from the points mentioned above, it can be concluded that image processing is a first step to change the image as desired by a certain method, either with the lightening and sharpness method used or with other methods; the method used is very influential on the output image. Or it could be said that the resulting image after applying the method. From the method that the author has applied, it can produce an image output that has image quality with sharpness and brightness following the level of sharpness and brightness applied by the system user.

The suggestions that the authors put forward in the preparation of this thesis are as follows:

- To produce an output image with more satisfactory quality, the authors suggest that the brightness and sharpness methods be applied by using other programming languages.

- From the research conducted by the author, there are several shortcomings in applying the brightness and sharpness method to the input image so that the resulting output images are less than optimal; therefore, the author strongly recommends that further researchers use this method of brightness and sharpness, applied to the application package with maximum process logic in memory allocation.

Lack of knowledge, the writer, can examine the performance and logic in this research. The writer said that it is necessary to develop the logic applied in processing RGB values of images to become high-quality output images.

\section{Acknowledgement}

Thank you very much to Almuslim University and Informatics Engineering, who have supported this research to be carried out. Hopefully, the research can help and contribute to the next researchers. This research was carried out with the support of the Almuslim University research institute.

\section{References}

[1] Y. Hendriana and R. Hardi, "Remote control system as serial communications mobile using a microcontroller," 2017, doi: 10.1109/ICITSI.2016.7858212.

[2] J. Febrian Rusdi et al., "ICT Research in Indonesia," 2019.

[3] R. Ajami and N. Al-Qirim, "IT Governance in Higher Education Institutions in Abu Dhabi, UAE," Int. J. It/bus. Alignment Gov., 2014, doi: 10.4018/ijitbag.2013070101.

[4] Budi Sunaryo et al., "Mapping Mining Potential Using WebGIS," SciTech Framew., vol. 1, no. 1, pp. 41-46, 2019.

[5] A. Rip, "Technology Assessment," in International Encyclopedia of the Social \& Behavioral Sciences: Second Edition, 2015.

[6] K. Patil and M. S. Kulkarni, "Artificial intelligence in financial services: Customer chatbot advisor adoption," Int. J. Innov. Technol. Explor. Eng., 2019, doi: 10.35940/ijitee.A4928.119119.

[7] A. Dwi Susandi, C. Sa'Dijah, A. Rahman As'Ari, and Susiswo, "Students' critical ability of mathematics based on cognitive styles," J. Phys. Conf. Ser., vol. 1315, no. 1, 2019, doi: 10.1088/1742-6596/1315/1/012018.

[8] "Service quality, customer satisfaction in technology-based universities," African J. Bus. Manag., 2011, doi: 10.5897/AJBM10.1687.

[9] S. J. Kwon, Artificial neural networks. 2011.

[10] R. Hardi, "Genetic algorithm in solving the TSP on these mineral water," 2015, doi: 10.1109/ISITIA.2015.7220008.

[11] A. K. Tiwari, "Introduction to machine learning," Ubiquitous Machine Learning and Its Applications. 2017, doi: 10.4018/978-1-5225-25455.ch001. 